TRANSACTIONS OF THE

AMERICAN MATHEMATICAL SOCIETY

Volume 350, Number 12, December 1998, Pages 4897-4918

S 0002-9947(98)02269-7

\title{
TWO DIMENSIONAL ELLIPTIC EQUATION WITH CRITICAL NONLINEAR GROWTH
}

\author{
TAKAYOSHI OGAWA AND TAKASHI SUZUKI
}

ABstract. We study the asymptotic behavior of solutions to a semilinear elliptic equation associated with the critical nonlinear growth in two dimensions.

$$
\left\{\begin{array}{cc}
-\Delta u=\lambda u e^{u^{2}}, u>0 & \text { in } \Omega, \\
u=0 & \text { on } \partial \Omega,
\end{array}\right.
$$

where $\Omega$ is a unit disk in $\mathbb{R}^{2}$ and $\lambda$ denotes a positive parameter.

We show that for a radially symmetric solution of (1.1) satisfies

$$
\int_{D}|\nabla u|^{2} d x \rightarrow 4 \pi, \quad \lambda \searrow 0
$$

Moreover, by using the Pohozaev identity to the rescaled equation, we show that for any finite energy radially symmetric solutions to (1.1), there is a rescaled asymptotics such as

$$
u_{m}^{2}\left(\gamma_{m} x\right)-u_{m}^{2}\left(\gamma_{m}\right) \rightarrow 2 \log \frac{2}{1+|x|^{2}} \quad \text { as } \lambda_{m} \searrow 0
$$

locally uniformly in $x \in \mathbb{R}^{2}$.

We also show some extensions of the above results for general two dimensional domains.

\section{IntRoduction}

The purpose of this paper is to study the asymptotic behavior of solutions of a semi-linear elliptic eigenvalue problem associated with critical nonlinear growth in two dimensions.

Let $\Omega \subset \mathbb{R}^{2}$ be a simply connected bounded domain with smooth boundary $\partial \Omega$. We consider the boundary value problem

$$
\left\{\begin{array}{cc}
-\Delta u=\lambda u e^{u^{2}}, u>0 & \text { in } \Omega, \\
u=0 & \text { on } \partial \Omega,
\end{array}\right.
$$

where $\lambda$ denotes a positive parameter.

This problem is related to a variational formulation connected with a Sobolev type inequality. In [24], N.S. Trudinger proved the existence of positive constants $a$ and $C_{0}$ such that

$$
\int_{\Omega}\left\{\exp \left(a v^{2}\right)-1\right\} d x \leq C_{0}|\Omega|
$$

Received by the editors January 29, 1996.

1991 Mathematics Subject Classification. Primary 35J60, 35P30, 35J20.

The first author is on long-term leave from the Graduate School of Polymathematics, Nagoya University, Nagoya 464-01 Japan. 
for any $v \in H_{0}^{1}(\Omega)$ with $\|\nabla v\|_{2} \leq 1$. Here and henceforth, $H_{0}^{1}(\Omega)=W_{0}^{1,2}(\Omega)$ denotes the Sobolev space equipped with the norm $\|\cdot\|_{H^{1}} \equiv\|\cdot\|_{2}+\|\nabla \cdot\|_{2}$.

Later, J. Moser [13] refined (1.2) and obtained a sharp range for $a$. That is, if $a \leq 4 \pi,(1.2)$ is valid and if $a>4 \pi,(1.2)$ does not hold. T. Ogawa [15] proposed a form valid for a general unbounded domain. Those results may be regarded as two dimensional versions of the Sobolev inequality,

$$
\|v\|_{\frac{2 n}{n-2}} \leq S_{0}(n)\|\nabla v\|_{2}
$$

with $n>2$ standing for the dimension in consideration. The striking difference in comparing to the higher dimensional case is that the extremal function of this inequality exists. This fact was first proved by L. Carleson and S.-Y.A. Chang [4] for the radially symmetric case, and later by M. Flucher [7] for general bounded domains. Then, the extremal function satisfies (1.1) in a weak sense with $\lambda$ as the associated Lagrange multiplier.

Concerning the equation (1.1) itself, B. McLeod and L.A. Peletier [12] gave a fine analysis based on the method of ordinary differential equation in the radially symmetric case. On the other hand, M.-C. Shaw [21] and Adimurthi [1] made use of variational methods to obtain solutions in bounded domains. Among other things, Adimurthi constructed positive solutions for any $\lambda$ with $0<\lambda<\lambda_{1}$, where $\lambda_{1}$ denotes the first eigenvalue of $-\Delta$ in $\Omega$ with homogeneous Dirichlet boundary condition. As we shall show later, those solutions are smooth.

In connection with those existence and smoothness results, it should be noted that classical solutions arise only when $\lambda>0$ by the maximal principle. On the other hand, multiplying by the first eigenfunction $\phi_{1}>0$ of the Laplacian, we realize that

$$
\lambda_{1} \int_{\Omega} u \phi_{1} d x=\lambda \int_{\Omega} u \phi_{1} e^{u^{2}} d x>\lambda \int_{\Omega} u \phi_{1} d x
$$

Therefore the existence of a classical solution also implies $\lambda<\lambda_{1}$.

In the present paper, we take different views from those works, particularly on the asymptotic behavior of solutions. First, any smooth solutions must blow up in $L^{\infty}$ norm as $\lambda \searrow 0$. In fact, multiplying the equation by $u$ and integrating by parts, we have

$$
\|\nabla u\|_{2}^{2}=\lambda \int_{\Omega} u^{2} e^{u^{2}} d x>0 .
$$

Therefore, by Poincaré's inequality, we deduce that

$$
\frac{1}{\lambda}=\frac{1}{\|\nabla u\|_{2}^{2}} \int_{\Omega} u^{2} e^{u^{2}} d x \leq e^{\|u\|_{\infty}^{2}} \frac{\|u\|_{2}^{2}}{\|\nabla u\|_{2}^{2}} \leq \frac{1}{\lambda_{1}} e^{\|u\|_{\infty}^{2}} \rightarrow \infty
$$

as $\lambda \searrow 0$.

According to the symmetry result by B. Gidas, Ni W.-M. and L. Nirenberg [8], any positive smooth solution is necessarily radially symmetric and decreasing with respect to $\rho=|x|$ on the unit disk. Then we have already shown the following ([16], $[17])$. 
Proposition 1.1. Any family of smooth solutions $\{(\lambda, u)\}$ of (1.1) on $\Omega=D \equiv$ $\left\{x \in \mathbb{R}^{2}|| x \mid=\rho<1\right\}$ satisfies the following conditions:

$$
\begin{aligned}
& \lim _{\lambda \rightarrow 0} u(x)=0 \quad \text { locally uniformly in } x \in \bar{D} \backslash\{0\}, \\
& \lim _{\lambda \rightarrow 0} \lambda \int_{D} u e^{u^{2}} d x=0, \\
& \lim _{\lambda \rightarrow 0} \lambda \int_{D}\left(e^{u^{2}}-1\right) d x=0, \\
& \varliminf_{\lambda \rightarrow 0} \int_{D}|\nabla u|^{2} d x \geq 4 \pi .
\end{aligned}
$$

In this paper, we shall prove the following for the radially symmetric solution.

Theorem 1.2. The family of solutions $\mathcal{C}=\{(u, \lambda)\}$ forms a one dimensional manifold in $C^{0}(\bar{D}) \times \mathbb{R}$. Furthermore,

$$
\int_{D}|\nabla u|^{2} d x \rightarrow 4 \pi
$$

as $\lambda \searrow 0$.

The above theorem, combined with the following result, yields the microscopic asymptotics.

Theorem 1.3. For any sequence $\left\{\left(u_{m}, \lambda_{m}\right)\right\}$ of smooth radially symmetric solutions with

$$
E_{0}=\varlimsup_{\lambda \rightarrow 0} \int_{D}\left|\nabla u_{m}\right|^{2} d x<\infty
$$

there are scaling parameters $\gamma_{m} \searrow 0$ as $\lambda_{m} \searrow 0$ satisfying

$$
u_{m}^{2}\left(\gamma_{m} x\right)-u_{m}^{2}\left(\gamma_{m}\right) \rightarrow 2 \log \frac{2}{1+|x|^{2}} \text { as } \lambda_{m} \searrow 0
$$

locally uniformly in $x \in \mathbb{R}^{2}$. Furthermore, we have

$$
\lambda_{m} \gamma_{m}^{2} u_{m}^{2}\left(\gamma_{m}\right) e^{u_{m}^{2}\left(\gamma_{m}\right)} \rightarrow 1
$$

Since the scaling parameter $\gamma_{m}$ is taken such that $u_{m}^{2}\left(\gamma_{m}\right) \rightarrow \infty$, the left-hand side in (1.8) represents a sort of scaling process corresponding to $\gamma_{m}^{(n-2) / 2} u\left(\gamma_{m} x\right)$ in higher dimensional cases $(n>2)$, while the limiting function of right-hand side is nothing but the unique solution of

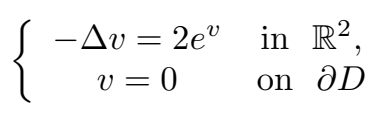

(cf. W.-X. Chen and C.-M. Li [5]). L. Carleson and S.-Y.A. Chang [4] implicitly pointed out this kind of asymptotics, while M. Struwe [23] obtained the analogous behavior of non-compact maximizing sequences for (1.2). Theorem 1.3 states that the same phenomenon also occurs in the eigenvalue problem (1.1). In fact, the asymptotics (1.8) is seen in somewhat weaker statements in [17] and [18]. We shall give a more general version of Theorem 1.3 in a general setting which is valid for any radial solutions.

We proceed to the case of a simply connected bounded domain. Existence of a family of solutions $\left\{\left(u_{m}, \lambda_{m}\right)\right\}$, is assured by the result of Adimurthi [1] based on the Nehari critical point theory [14]. 
Proposition $1.4([1])$. For $0<\lambda<\lambda_{1}$, there is a weak solution $u$ to (1.1) and it becomes regular, i.e. $u \in C^{\infty}(\Omega)$.

Besides this formulation, there are several existence results using the variational method (M. Flucher [7], M.-C. Shaw [21]). We shall discuss on their relations between those variational solutions as well as their regularity.

For those solutions, we can extend Proposition 1.1 and Theorem 1.2 in the following way.

Define the blow-up set by

$$
\begin{aligned}
& \mathcal{S}=\left\{x \in \bar{\Omega} \quad \mid \quad \text { there exist sequences } \quad x_{m_{j}} \rightarrow x \text { and } \lambda_{m_{j}} \searrow 0\right. \\
& \text { such that } u_{m_{j}}\left(x_{m_{j}}\right) \rightarrow \infty \text { as } j \rightarrow \infty \text { \}. }
\end{aligned}
$$

Theorem 1.5. Given a family $\{(u, \lambda)\}$ of smooth solutions of (1.1), one has

$$
\begin{aligned}
& \lim _{\lambda \rightarrow 0}\|u\|_{\infty}=+\infty, \\
& \lim _{\lambda \rightarrow 0} \lambda \int_{\Omega} u e^{u^{2}} d x=0, \\
& \lim _{\lambda \rightarrow 0} \lambda \int_{\Omega}\left(e^{u^{2}}-1\right) d x=0 .
\end{aligned}
$$

If

$$
\varlimsup_{\lambda \rightarrow 0} \int_{\Omega}|\nabla u|^{2} d x<\infty,
$$

then for each $x \in \mathcal{S}$ and $\delta>0$ with $D_{\delta}(x) \equiv\{y \in \Omega|| x-y \mid<\delta\} \subset \Omega$ one has the estimate

$$
\varliminf_{\lambda \rightarrow 0} \int_{D_{\delta}(x)}|\nabla u|^{2} d x \geq 4 \pi
$$

and furthermore,

$$
u(x) \rightarrow 0 \text { locally uniformly on } \bar{\Omega} \backslash \mathcal{S}
$$

as $\lambda \searrow 0$.

Consequently, we have

$$
\# \mathcal{S} \leq \frac{1}{4 \pi} \varliminf_{\lambda \rightarrow 0} \int_{\Omega}|\nabla u|^{2} d x .
$$

Analogous facts for higher dimensional cases have been seen (e.g., Itoh [10]).

As for the variational solution, we have the following.

Proposition 1.6. Let $\Omega$ be a simply connected bounded domain in $\mathbb{R}^{2}$. Then the family of solutions $\{(u, \lambda)\}$ of (1.1) obtained by Adimurthi [1] satisfies

$$
\lim _{\lambda \rightarrow 0}\|\nabla u\|_{2}^{2}=4 \pi \text {. }
$$

Hence the blow-up set $\mathcal{S}$ consists of one point of $\Omega$.

Putting $\mathcal{S}=\left\{x_{0}\right\}$, we have

$$
|\nabla u|^{2} d x \rightarrow 4 \pi \delta_{x_{0}}(x)
$$

in the sense of measures for this kind of solution.

This paper is divided into five sections. Section 2 is devoted to (1.1) on the unit ball. We shall prove Theorem 1.2. Section 3 concerns the asymptotics of Theorem 
1.3 for a general nonlinearity on the unit ball, and Theorem 4 of our previous paper [18] is improved (Theorem 3.1). Also the non-uniformity around $x=\gamma_{m}^{-1}$ of (1.8) is stated. In $\S 4$, we examine various variational solutions. The regularity of a weak solution to (1.1) will be discussed. Also the relationship between the several variational formulations will be clarified (Propositions 4.1, 4.6, 4.7). Finally, §5 treats simply connected domains. In particular, Theorem 1.5 and Proposition 1.6 are proved.

\section{Equation on the Unit Disk}

This section is mostly devoted to the proof of Theorem 1.2. Hence $u=u(r)$ denotes the solution of (1.1) on $\Omega=D$.

Proof of Theorem 1.2. Introducing the transformation $y(\tau)=u(r)$ with $\tau=$ $-2 \log r-\log \frac{\lambda}{4}$ to eliminate the parameter $\lambda$, we transform the original problem into the ordinary differential equation

$$
y^{\prime \prime}+y e^{y^{2}-\tau}=0 \quad(-\infty<\tau<\infty)
$$

with $y^{\prime}>0(\tau>0)$ and

$$
\begin{aligned}
& y(\tau)>0 \text { for } \tau>\tau_{0}, \\
& y\left(\tau_{0}\right)=0, \\
& \lim _{\tau \rightarrow \infty} y(\tau)=y(\infty) \equiv \zeta .
\end{aligned}
$$

Here the parameter $\lambda>0$ is transformed into the first (largest) zero hit point $\tau_{0}$ of $y(\tau)$ by $\tau_{0}=-\log \frac{\lambda}{4}$, and $x=0$ is transformed into $\tau=\infty$. The solution set $\mathcal{C}=\{(u, \lambda)\}$ has been parameterized by $\zeta \in(0, \infty)$. Furthermore, since each zero of $y$ is non-degenerate because $y^{\prime} \neq 0$ at each zero, the mapping $\zeta \mapsto \tau_{0}$ is differentiable. Hence $\mathcal{C}$ forms a smooth curve in $C^{0}(\bar{D}) \times \mathbb{R}$, and the first part of Theorem 1.2 has been proved.

Next we show that $\|\nabla u\|_{2}^{2} \rightarrow 4 \pi$ as $\lambda \searrow 0$. As is indicated in (1.7), the inequality

$$
\varliminf_{\lambda \rightarrow 0}\|\nabla u\|_{2}^{2} \geq 4 \pi
$$

follows from the Trudinger-Moser inequality. In fact, if this is not the case, (1.2) with $a=4 \pi$ implies $\left\|e^{(1+\varepsilon) u^{2}}\right\|_{1}=O(1)$ for a subsequence. Then, a standard elliptic estimate (e.g., Gilbarg-Trudinger [9], Theorem 8.15) implies that $\|u\|_{\infty} \rightarrow 0$ as $\lambda \searrow 0$, which yields a contradiction. It then suffices to show that

$$
\varlimsup_{\lambda \rightarrow 0} \int_{D}|\nabla u|^{2} d x=\varlimsup_{\lambda \rightarrow 0} \int_{\tau_{0}}^{\infty} y^{\prime}(\tau)^{2} d \tau \leq 1
$$

in the transformed variable $\tau=-2 \log r+\tau_{0}$.

Equation (2.1) is free from parameters, and has been studied by J. B. McLoad and L.A. Peletier [12] precisely. We shall make use of the following asymptotic formulae as $\zeta \rightarrow+\infty$.

1. ([12, Lemma 4]) We have

$$
\tau_{0}=2 \log \zeta+1+o(1)
$$

2. (p.273 in [12]) Let

$$
H(\tau)=y^{2}(\tau)+2 \log y(\tau)-\tau
$$


and let $\tau^{*}$ be its first zero as $\tau$ decreases from $+\infty$. Then

$$
y\left(\tau^{*}\right)=\zeta+O\left(\zeta^{-1}\right)
$$

and

Consequently,

$$
e^{H\left(\tau^{*}\right)}=\frac{1}{4}+o(1)
$$

$$
\begin{aligned}
y^{\prime}\left(\tau^{*}\right) & =\frac{1}{2} \cdot \frac{y\left(\tau^{*}\right)}{y\left(\tau^{*}\right)^{2}+1}\left(H^{\prime}\left(\tau^{*}\right)+1\right) \\
& =\frac{1}{2} \zeta^{-1}\{1+o(1)\}\left(H^{\prime}\left(\tau^{*}\right)+1\right)
\end{aligned}
$$

3. ((6.4) in [12]) We have

$$
\tau^{*}=\zeta^{2}+2 \log \zeta+O(1)
$$

(precise expression of (6.2)). Hence

$$
\tau^{*}-\tau_{0}=\zeta^{2}+O(1)
$$

and

$$
\tau_{2}-\tau_{0}=\zeta^{2}\{1+o(1)\},
$$

where $\tau_{2}=\tau^{*}-k \log \zeta$ with $k>2$.

4. ((6.13) and p.278 in [12]) We have

$$
y^{\prime}(\tau)=\zeta^{-1}\{1+o(1)\} \quad\left(\tau_{0} \leq \tau \leq \tau_{2}\right) .
$$

5. (p.273 in [12]) We have

$$
H^{\prime}+1=2 y^{-1} y^{\prime}\left(y^{2}+1\right) \approx e^{H}=y^{2} e^{y^{2}-\tau} \quad\left(\tau \geq \tau^{*}\right),
$$

where $\alpha \approx \beta$ indicates the existence of a constant $K>0$ satisfying

$$
K^{-1}|\alpha| \leq|\beta| \leq K|\alpha|
$$

This implies that

$$
y^{\prime} \approx y e^{y^{2}-\tau} \quad\left(\tau \geq \tau^{*}\right) .
$$

6. The concavity of $y$, i.e., $y^{\prime \prime}<0,(2.5)$ and (2.8) imply that for $\tau_{2} \leq \tau \leq \tau^{*}$,

$$
\frac{1}{2} \zeta^{-1}\left(H^{\prime}\left(\tau^{*}\right)+1\right)\{1+o(1)\} \leq y^{\prime}(\tau) \leq \zeta^{-1}\{1+o(1)\} .
$$

Admitting them, we can show (2.3) in the following way.

Proof of (2.3). First, we have from (2.7) and (2.8) that

$$
\int_{\tau_{0}}^{\tau_{2}} y^{\prime}(\tau)^{2} d \tau=\zeta^{2}\{1+o(1)\} \cdot \zeta^{-2}\{1+o(1)\}=1+o(1) .
$$

Next, (2.10) implies

$$
\begin{aligned}
\int_{\tau_{2}}^{\tau^{*}} y^{\prime}(\tau)^{2} d \tau & \leq\left(\tau^{*}-\tau_{2}\right) \zeta^{-2}\{1+o(1)\} \\
& =(k \log \zeta) \zeta^{-2}\{1+o(1)\}=o\left(\zeta^{-1}\right) .
\end{aligned}
$$

Finally, since the relations (2.9) and (2.4) imply

$$
y^{\prime}(\tau) \approx e^{-\tau_{0}} y e^{y^{2}-\tau} \approx e^{-1} \zeta^{-2} y e^{y^{2}-\tau} \quad\left(\tau \geq \tau^{*}\right),
$$


it follows from $y(\tau) \leq y(\infty)=\zeta$ and (2.11) that

$$
\begin{aligned}
\int_{\tau^{*}}^{\infty} y^{\prime}(\tau)^{2} d \tau & \approx e^{-2} \zeta^{-4} \int_{\tau^{*}}^{\infty} y^{2}(\tau) e^{2\left(y^{2}(\tau)-\tau\right)} d \tau \\
& \leq e^{-2} \zeta^{-2} e^{2 \zeta^{2}} \int_{\tau^{*}}^{\infty} e^{-2 \tau} d \tau \\
& =\frac{1}{2 e^{2}} \zeta^{-2} e^{2 \zeta^{2}-2 \tau^{*}}
\end{aligned}
$$

However, from (2.6),

$$
\zeta^{2}-\tau^{*}=-\tau_{0}+O(1)
$$

and the last term in (2.12) disappears as $\zeta \rightarrow+\infty$.

In this way, (2.3) has been proved.

\section{Microscopic Behavior for Radial Solutions}

3.1. Proof of Theorem $\mathbf{1 . 3}$ for general nonlinearity. This section is devoted to establishing the asymptotic behavior Theorem 1.3 under the general situation. Let $D$ be the two-dimensional unit ball. We shall treat the following problem

$$
\left\{\begin{array}{cc}
-\Delta u=\lambda f(u) e^{u^{2}}, u>0 & \text { in } D \\
u=0 & \text { on } \partial D
\end{array}\right.
$$

The nonlinearity $f(u)$ satisfies $f \in C^{1}$,

$$
f(u), f^{\prime}(u) \geq 0(u \geq 0)
$$

and, for $g(u)=u f(u)$,

$$
(\log g)^{\prime}(u) \leq M(u \gg 1),
$$

where $M>0$ is a constant. We assume the existence of a smooth solution such that $u(0) \rightarrow \infty$ as $\lambda \searrow 0$. In fact, in a certain case, we have the existence of such solutions, as we have seen in the first section.

The following theorem is an improvement of Theorem 4 of [18].

Theorem 3.1. Suppose that $\left\{u_{m}, \lambda_{m}\right\}$ is a family of solutions to (3.1) satisfying

$$
E_{m}=\int_{D}\left|\nabla u_{m}\right|^{2} d x<\infty .
$$

Then for some scaling parameter $\gamma_{m} \searrow 0$, we have

$$
u_{m}^{2}\left(\gamma_{m} x\right)-u_{m}^{2}\left(\gamma_{m}\right) \rightarrow 2 \log \frac{2}{1+|x|^{2}} \text { as } \lambda_{m} \searrow 0
$$

locally uniformly in $x \in \mathbb{R}^{2}$.

Proof of Theorem 3.1. The proof is divided into several steps. First we recall the following fact for (3.1), proved in our previous work [17].

Lemma 3.2 ([17]). Let $\{(u, \lambda)\}$ be any family of solutions of (3.1). Then we have

$$
\rho u^{\prime}(\rho) \rightarrow 0 \quad \text { uniformly on } \bar{D} \text { as } \lambda \searrow 0 \text {, }
$$

where $\rho=|x|$. In particular,

$$
u(\rho) \rightarrow 0 \text { locally uniformly on } \bar{D} \backslash\{0\} .
$$


Henceforth, taking a subsequence $\left\{\left(u_{\lambda_{m}}, \lambda_{m}\right)\right\}$ of $\{(u, \lambda)\}$, we omit their subscripts. Following [17], we define the rescaled solution $v$ by

$$
v(r)=u^{2}(\gamma r)-u^{2}(\gamma)
$$

where $\gamma>0$ is a scaling constant to be determined later. Then, $v$ solves the equation

$$
\left\{\begin{array}{l}
-\Delta v=2 k(r) e^{v}-2 \gamma^{2} u_{\rho}^{2}, \\
v(1)=0
\end{array}\right.
$$

in $0 \leq r<\gamma^{-1}$ for $r=\gamma^{-1} \rho$, where

$$
k(r)=\lambda \gamma^{2} e^{u(\gamma)^{2}} u(\gamma r) f(u(\gamma r))
$$

and $u_{\rho}=\left.\partial_{\rho} u(\rho)\right|_{\rho=\gamma r}$. Furthermore, $v_{r}(r) \leq 0$ and $k_{r}(r) \leq 0$.

We choose the scaling parameter $\gamma$, which depends on $\lambda$, such that

$$
u^{2}(0)-u^{2}(\gamma)=2 \log 2 .
$$

Then since $u(0) \rightarrow \infty$ as $\lambda \rightarrow 0,(3.6)$ and (3.9) imply that $\gamma \rightarrow 0$ and

$$
\|v\|_{L^{\infty}(D)} \leq 2 \log 2 .
$$

Furthermore, (3.5) in Lemma 3.2 yields

$$
\begin{aligned}
\gamma^{2} u_{\rho}^{2}(\gamma r) & =(\gamma r)^{2} u_{\rho}^{2}(\gamma r) / r^{2} \\
& \rightarrow 0 \quad \text { locally uniformly in } r \in(0, \infty) .
\end{aligned}
$$

We shall present two identities for the rescaled equation (3.8). Corresponding ones for the original equation (3.1) have been utilized for the macroscopic asymptotics, that is, the formulae driven by Gauss' lemma and Pohozaev's identity ([17]).

Lemma 3.3. For any $0 \leq a<1$,

$$
-v_{r}(1)+a v_{r}(a)=2 \int_{a}^{1} k(r) r e^{v} d r-2 \int_{a}^{1} \gamma^{2} r u_{\rho}^{2}(\gamma r) d r
$$

and

$$
\begin{aligned}
-\frac{1}{2} v_{r}(1)^{2}+ & \frac{1}{2} a^{2} v_{r}(a)^{2}=2 k(1)-2 a^{2} k(a) e^{v(a)}-4 \int_{a}^{1} r k(r) e^{v(r)} d r \\
& -2 \int_{a}^{1} r^{2} k_{r}(r) e^{v(r)} d r-2 \int_{a}^{1} \gamma^{2} r^{2} u_{\rho}^{2}(\gamma r) v_{r}(r) d r .
\end{aligned}
$$

Proof. The equation (3.12) follows from multiplying

$$
-\frac{1}{r}\left(r v_{r}\right)_{r}=2 k(r) e^{v(r)}-2 \gamma^{2} u_{\rho}^{2}(\gamma r)
$$

by $r$ with integration over $(a, 1)$. 
For (3.13), we multiply (3.14) by $r^{2} v_{r}$, and integrate by parts over $(a, 1)$. Then

$$
\begin{aligned}
-\frac{1}{2} v_{r}(1)^{2}+ & \frac{1}{2} a^{2} v_{r}(a)^{2} \\
= & 2 \int_{a}^{1} r^{2} k(r) e^{v(r)} v_{r}(r) d r-2 \int_{a}^{1} \gamma^{2} r^{2} u_{\rho}^{2}(\gamma r) v_{r}(r) d r \\
= & 2 k(1)-2 a^{2} k(a) e^{v(a)}-4 \int_{a}^{1} r k(r) e^{v(r)} d r \\
& -2 \int_{a}^{1} r^{2} k_{r}(r) e^{v(r)} d r-2 \int_{a}^{1} \gamma^{2} r^{2} u_{\rho}^{2}(\gamma r) v_{r}(r) d r
\end{aligned}
$$

and hence (3.13).

In the rest of the present section, we utilize the condition

$$
\varlimsup_{\lambda \rightarrow 0} \int_{D}|\nabla u|^{2} d x=E_{0}<+\infty .
$$

We prove the following.

Lemma 3.4. There exists a constant $C\left(E_{0}\right)>0$, independent of $\lambda \in\left(0, \lambda_{1}\right)$, such that

$$
0 \leq K \equiv k(1) \leq C\left(E_{0}\right)
$$

Proof. Since $v_{r}(r) \leq 0$ and $v \geq 0(0 \leq r<1)$, by $(3.2)$ we have $k_{r}(r) \leq 0$ on $[0,1)$ in Lemma 3.3. Then, choosing $a=1$ in (3.12) and (3.13),

$$
\begin{aligned}
\frac{1}{2} v_{r}^{2}(1)= & -2 K+4 \int_{0}^{1} r k(r) e^{v(r)} d r \\
& +2 \int_{0}^{1} r^{2} k_{r}(r) e^{v(r)} d r+2 \int_{0}^{1} \gamma^{2} r^{2} u_{\rho}^{2}(\gamma r) v_{r}(r) d r \\
\leq & 4 \int_{0}^{1} r k(r) e^{v(r)} d r \\
= & -2 v_{r}(1)+4 \int_{0}^{1} \gamma^{2} u_{\rho}^{2}(\gamma r) r d r \\
\leq & -2 v_{r}(1)+\frac{2}{\pi} \int_{B}|\nabla u|^{2} d x
\end{aligned}
$$

Therefore, $-v_{r}(1) \leq C^{\prime}\left(E_{0}\right)$, and hence

$$
\int_{0}^{1} r k(r) e^{v(r)} d r \leq C^{\prime \prime}\left(E_{0}\right)
$$

where $C^{\prime}\left(E_{0}\right)$ and $C^{\prime \prime}\left(E_{0}\right)$ are constants determined by $E_{0}$.

From the first equality of (3.16), we conclude that

$$
K \leq 2 \int_{0}^{1} r k(r) e^{v(r)} d r \leq C\left(E_{0}\right)
$$

According to (3.15) in Lemma 3.4, there exist two constants $\mu_{+} \geq \mu_{-} \geq 0$ such that

$$
\mu_{+}=\varlimsup_{\lambda \rightarrow 0} K \quad \text { and } \quad \mu_{-}=\varliminf_{\lambda \rightarrow 0} K .
$$


Then we can extract two subsequences $\left\{u_{+}\right\}$and $\left\{u_{-}\right\}$such that

$$
k^{+}(1) \equiv \lambda \gamma^{2} e^{u_{+}(\gamma)^{2}} u_{+}(\gamma) f\left(u_{+}(\gamma)\right) \rightarrow \mu_{+}
$$

and

$$
k^{-}(1) \equiv \lambda \gamma^{2} e^{u_{-}(\gamma)^{2}} u_{-}(\gamma) f\left(u_{-}(\gamma)\right) \rightarrow \mu_{-} .
$$

We have the following estimates for those sequences.

Lemma 3.5. For any subsequence $\{K=k(1)\}$ converging to some constant $\mu$ $\left(\mu_{-} \leq \mu \leq \mu_{+}\right)$, the corresponding sequence $\{k(r)\}$ satisfies

$$
\mu \min \left(r^{-\eta M}, 1\right)+o(\lambda) \leq k(r) \leq \mu \max \left(r^{-\eta M}, 1\right)+o(\lambda),
$$

where $\eta \searrow 0$ as $\lambda \searrow 0$. Therefore

$$
k(r) \rightarrow \mu \quad \text { locally uniformly in } r \in(0, \infty)
$$

as $\lambda \searrow 0$.

Proof. We recall that $g(u)=u f(u)$. First we take $r$ in $0<r<1$. Since $r \mapsto u(\gamma r)$ is decreasing, we see that

$$
\begin{aligned}
1 & \leq \frac{g(u(\gamma r))}{g(u(\gamma))}=1-\frac{1}{g(u(\gamma))} \int_{r}^{1} \gamma g^{\prime}(u(\gamma s)) u_{\rho}(\gamma s) d s \\
& =1-\int_{r}^{1} \frac{g(u(\gamma s))}{g(u(\gamma))} \cdot \frac{g^{\prime}(u(\gamma s))}{g(u(\gamma s))} \cdot \gamma s u_{\rho}(\gamma s) \frac{d s}{s} .
\end{aligned}
$$

Hence by (3.3), Lemma 3.2 and the fact that $u(\gamma) \rightarrow+\infty$ by (3.9), we have for any small $\eta>0$ that

$$
X(r) \equiv \frac{g(u(\gamma r))}{g(u(\gamma))} \leq 1+\eta M \int_{r}^{1} X(s) \frac{d s}{s} \equiv F(r)
$$

for small $\lambda$. Then

$$
F^{\prime}(r)=-\eta M \frac{X(r)}{r} \geq-\eta M \frac{F(r)}{r},
$$

which yields that

$$
1 \leq X(r) \leq r^{-\eta M}, \quad r \in(0,1]
$$

Hence

$$
\begin{aligned}
\mu+o(\lambda) \leq k(r) & =\frac{g(u(\gamma r))}{g(u(\gamma))} k(1) \\
& \leq \mu r^{-\eta M}+o(\lambda) \quad \text { on }(0,1] .
\end{aligned}
$$

In the similar way, we have

$$
r^{-\eta M} \leq X(r) \leq 1, \quad r \in[1, \infty) .
$$

Therefore we obtain

$$
\mu \min \left(r^{-\eta M}, 1\right)+o(\lambda) \leq k(r) \leq \mu \max \left(r^{-\eta M}, 1\right)+o(\lambda),
$$

and $k(r)$ converges $\mu$ locally uniformly in $r \in(0, \infty)$ as $\lambda \searrow 0$. Hence the conclusion follows.

The normalization (3.9) implies that $\mu_{+}=\mu_{-}=1$ and prescribes the limiting function of the rescaled family $\{v\}$. To see this, let $\left\{v^{ \pm}\right\}$be the subsequences defined by

$$
v^{ \pm}(r)=u_{ \pm}^{2}(\gamma r)-u_{ \pm}^{2}(\gamma)
$$


Lemma 3.6. There exist smooth functions $v_{0}^{ \pm} \in C(D) \cap C^{2}\left(\mathbb{R}^{2} \backslash\{0\}\right)$ satisfying, for some subsequence of $\left\{v^{ \pm}\right\}$,

Moreover, $v_{0}^{ \pm}$satisfies

$$
v^{ \pm}(r) \rightarrow v_{0}^{ \pm}(r) \quad \text { locally uniformly in }(0, \infty) .
$$

$$
\left\{\begin{array}{c}
-\Delta v_{0}^{ \pm}=2 \mu_{ \pm} e^{v_{0}^{ \pm}}, \quad x \in \mathbb{R}^{2} \backslash\{0\} \\
v_{0}^{ \pm}=0,
\end{array}\right.
$$

and

$$
\left\|v_{0}^{ \pm}\right\|_{L^{\infty}(D)} \leq 2 \log 2 .
$$

Proof. Similarly to [17], the above assertion is proved by Lemma 3.5 and the a priori estimate

$$
\left\|v^{ \pm}\right\|_{L^{\infty}(D)} \leq 2 \log 2 .
$$

In fact, (3.14) with (3.11) implies $\left\|\partial^{2} v^{ \pm}\right\|_{L^{\infty}(\omega)}$ is bounded independently of $\lambda$ for any $\omega \subset \subset \mathbb{R}^{2}$. The Ascoli-Arzela Theorem assures the existence of non-increasing $C^{1}$ functions $v_{0}^{ \pm}(r)$ such that

$$
v^{ \pm}(r) \rightarrow v_{0}^{ \pm}(r) \quad \text { locally uniformly in }(0, \infty),
$$

passing to subsequences. Now, each of $v_{0}^{ \pm}$becomes a classical solution for (3.18) subject to the estimate (3.10), because of (3.11) and (3.17).

Here is the crucial lemma.

Lemma 3.7. We have $\mu_{+}=\mu_{-}=1$ and

$$
v_{0}=v_{0}^{ \pm}=2 \log \frac{2}{1+r^{2}} .
$$

Proof. First, we verify that $\mu_{+} \leq 1$. For this purpose, we have only to take the case $\mu_{+}>0$. Changing the variable by $t=2 \log \frac{1}{r}$ and $w(t)=v_{0}^{+}(r)+2 \log r$ yields

$$
-w^{\prime \prime}(t)=\frac{\mu_{+}}{2} e^{w(t)}
$$

for $t \geq 0$, and hence

$$
w^{\prime}(t)^{2}+\mu_{+} e^{w(t)}=\alpha
$$

with some $\alpha \in \mathbb{R}$. The boundary condition $v^{+}(1)=0$ is equivalent to $w(0)=0$. Hence

$$
\alpha=w^{\prime}(0)^{2}+\mu_{+} \geq \mu_{+} .
$$

On the other hand, the equation for $w(t)$ is integrable and

$$
w^{\prime}(t)=\frac{\sqrt{\alpha}\left(1+A e^{\sqrt{\alpha} t}\right)}{1-A e^{\sqrt{\alpha} t}},
$$

where $A=2 \alpha-1 \pm \sqrt{(2 \alpha-1)^{2}-1}$. Therefore,

$$
0 \leq e^{w(t)}=\frac{1}{\mu_{+}}\left(\alpha-w^{\prime}(t)^{2}\right)=\frac{4 \alpha A}{\mu_{+}} \frac{e^{-\sqrt{\alpha} t}}{\left(A+e^{-\sqrt{\alpha} t}\right)^{2}} \approx e^{-\sqrt{\alpha} t},
$$

as $t \rightarrow \infty$. However, the positivity of $v^{+}(r)$ implies

$$
e^{w(t)}=r^{2} e^{v^{+}(r)} \geq r^{2}=e^{-t} .
$$

Hence $\alpha \leq 1$. This shows that $\mu_{+} \leq 1$. 
We next show that

$$
v_{0}^{-}(0)=\lim _{t \rightarrow 0} v_{0}^{-}(t)=2 \log 2 .
$$

Then the proof will be completed. In fact, this implies $\mu_{-}>0$. Now, smooth solutions for (3.18) are classified when $0<\mu \leq 1$. That is,

$$
v_{0}(r)=\log \left\{\frac{4 \beta_{ \pm}}{\mu\left(1+\beta_{ \pm} r^{2}\right)^{2}}\right\}
$$

where $\beta_{ \pm}=\frac{2}{\mu}\left\{1-\frac{\mu}{2} \pm \sqrt{1-\mu}\right\}$. Therefore, (3.20) will imply $\mu_{-}=1$ and $v_{0}^{ \pm}=v_{0}$.

The a priori bound for (3.19) admits a sequence $r_{n} \rightarrow 0$ such that

$$
r_{n} \log r_{n} \partial_{r} v_{0}^{-}\left(r_{n}\right) \rightarrow 0 .
$$

Otherwise, there would be a constant $C>0$ such that

$$
2 \log 2 \geq v_{0}^{-}(0)=-\int_{0}^{1} \partial_{r} v_{0}^{-}(r) d r \geq-C \int_{0}^{1} \frac{d r}{r \log r}=\infty,
$$

which is impossible.

For $s>0$, we have the relation

$$
\begin{aligned}
\int_{r_{n}}^{s} 2 \mu_{-} e^{v_{0}^{-}} r \log \frac{s}{r} d r & =-\int_{r_{n}}^{s} \Delta v_{0}^{-} r \log \frac{s}{r} d r \\
& =-\int_{r_{n}}^{s} \partial_{r}\left(r \partial_{r} v_{0}^{-}(r)\right) \log \frac{s}{r} d r \\
& =r_{n} \log \frac{s}{r_{n}} \partial_{r} v_{0}^{-}\left(r_{n}\right)-v_{0}^{-}(s)+v_{0}^{-}\left(r_{n}\right) .
\end{aligned}
$$

Hence

$$
2 \mu_{-} \int_{0}^{s} e^{v_{0}^{-}(r)} r \log \frac{s}{r} d r=-v_{0}^{-}(s)+v_{0}^{-}(0) .
$$

Similarly, (3.8) gives us

$$
2 \int_{0}^{s} \gamma^{2} u_{-\rho}^{2} r \log \frac{s}{r} d r=2 \int_{0}^{s} k_{-}(r) e^{v^{-}(r)} r \log \frac{s}{r} d r+v^{-}(s)-v^{-}(0) .
$$

Then the dominated convergence theorem yields

$$
\begin{aligned}
0 & \leq 2 \lim _{\lambda \rightarrow 0} \int_{0}^{s} \gamma^{2} u_{-\rho}^{2}(\gamma r) r \log \frac{s}{r} d r \\
& =2 \lim _{\lambda \rightarrow 0} \int_{0}^{s} k_{-}(r) e^{v^{-}(r)} r \log \frac{s}{r} d r+\lim _{\lambda \rightarrow 0}\left[v^{-}(s)-v^{-}(0)\right] \\
& =2 \mu_{-} \int_{0}^{s} e^{v_{0}^{-}(r)} r \log \frac{s}{r} d r+v_{0}^{-}(s)-2 \log 2 .
\end{aligned}
$$

Combining (3.21) and (3.23) with $v_{0}^{-}(0) \leq 2 \log 2$, we conclude that $v_{0}^{-}(0)=$ $2 \log 2$.

The above argument assures the convergence (3.4) of the rescaled solutions. The relation (1.9) is a consequence of $\mu_{+}=\mu_{-}=1$. Furthermore,

$$
\lim _{\lambda \rightarrow 0} \int_{0}^{s} \gamma^{2} u_{\rho}^{2}(\gamma r) r \log \frac{s}{r} d r=0 .
$$


Only the uniformity around $y=0$ of (3.4) is left for Theorem 3.1.

\section{Lemma 3.8.}

$$
v(r) \rightarrow 2 \log \frac{2}{1+r^{2}} \quad \text { locally uniformly on } \mathbb{R}^{2} .
$$

Proof. It suffices to show the convergence on $D$. Noting that $v_{0}(0)=v(0)=2 \log 2$, we subtract (3.22) from (3.21). We have, for $s=1$,

$$
\begin{aligned}
\left|v(s)-v_{0}(s)\right| \leq & \int_{0}^{s} \gamma^{2} u_{\rho}^{2}(\gamma r) r \log \frac{s}{r} d r \\
& +2 \int_{0}^{s}\left|k(r) e^{v(r)}-\mu e^{v_{0}(r)}\right| r \log \frac{s}{r} d r .
\end{aligned}
$$

The second term on the right hand side of (3.25) tends to zero by the dominated convergence theorem, while the first term also disappears by (3.24) as $\lambda \searrow 0$. This proves the desired consequence.

3.2. Non-uniformity of the asymptotics. Concluding the present section, we remark that for the simplest case $f(u)=u$, the uniform convergence of the asymptotics (3.4) does not hold for the solutions obtained by the ordinary differential equation approach (McLeod-Peletier [12]). That is, we have

Proposition 3.9. For a solution obtained by McLeod-Peletier [12] in the case (1.1) i.e., $f(u)=u$, the asymptotics (3.4) cannot be uniform on $\mathbb{R}^{2}$.

Proof. Suppose on the contrary that we could put $x=\gamma_{m}^{-1}$ in (3.4). For simplicity, we shall omit all subscripts $m$. Then, recalling that $u(1)=0$,

$$
0=u^{2}(\gamma)+2 \log \frac{2}{1+\gamma^{-2}}+o(1)
$$

We then choose $x=0$ in the right hand side of (3.4) to obtain

$$
u^{2}(0)=u^{2}(\gamma)+2 \log 2+o(1)
$$

Comparing (3.26) and (3.27), we have

$$
u^{2}(0)=2 \log \left(1+\gamma^{-2}\right)+o(1) .
$$

On the other hand, by $(2.4)$

$$
\lambda=4 e^{-\tau_{0}}=\frac{4}{e u^{2}(0)}\{1+o(1)\},
$$

where $\tau_{0}$ is the first zero for $y(\tau)$. Then, putting (3.29) into (1.9), we obtain

$$
u^{2}(\gamma)+2 \log \gamma+2 \log 2-1+2 \log \frac{u(\gamma)}{u(0)}=o(1) .
$$

Since by $(3.27)$

$$
\log \frac{u(\gamma)}{u(0)}=o(1)
$$

we see that

$$
u^{2}(\gamma)=1-2 \log 2-2 \log \gamma+o(1) .
$$

Again by (3.27), we have

$$
u^{2}(0)=1+\log \gamma^{-2}+o(1),
$$

which contradicts (3.28). 


\section{VARiational Solutions}

In the present section, we examine variational solutions on general domains. There are several formulation of the variational solution to (1.1). For example, by Adimurthi's method we can construct a weak solution of (1.1) by Nehari's critical point theory $([14])$. On the other hand, there is a result by M.-C. Shaw [21] in which a different kind of variational formulation is used. All those solutions satisfy the equation (1.1) in a weak sense. First we discuss the regularity of a weak solution to $(1.1)$.

4.1. Regularity of a weak solution. According to the result of H. Brezis and F. Merle [3], we may simply show that the weak solution to (1.1) is necessarily smooth. To see this, it suffices to show the following from elliptic regularity theory (e.g., Gilbarg-Trudinger [9], Chapter 8).

Proposition 4.1. Any weak solution to (1.1) belongs to $L^{\infty}(\Omega)$.

The following lemma due to H. Brezis and F. Merle [3] will be utilized for the proof.

Lemma 4.2 (Theorem 2.1 in [3]). For any fixed $\varepsilon>0$ let $v$ be a solution of

$$
\begin{cases}-\Delta v=f & \text { in } \Omega, \\ v=0 & \text { on } \partial \Omega,\end{cases}
$$

with $\|f\|_{1}<\varepsilon$. Then $e^{v} \in L^{4 \pi / \varepsilon}$.

Proof of Proposition 4.1. We recall the constraint (1.3) to note that $u^{2} e^{u^{2}} \in L^{1}$. Testing $u \phi$ in (1.1), we have

$$
\int_{\Omega} u \nabla u \cdot \nabla \phi d x=\lambda \int_{\Omega} u^{2} e^{u^{2}} \phi d x-\int_{\Omega}|\nabla u|^{2} \phi d x
$$

where $\phi \in C_{0}^{\infty}$. In other words, $u^{2} \in W^{1,1}$ solves

$$
\left\{\begin{array}{cc}
-\Delta u^{2}=2 \lambda u^{2} e^{u^{2}}-2|\nabla u|^{2} & \text { in } \Omega, \\
u^{2}=0 & \text { on } \partial \Omega
\end{array}\right.
$$

in the weak sense.

The problem

$$
\left\{\begin{array}{cc}
-\Delta h=-2|\nabla u|^{2} & \text { in } \Omega, \\
h=0 & \text { on } \partial \Omega
\end{array}\right.
$$

is uniquely solvable. From the maximum principle, $h \leq 0$ a.e. in $\Omega$. We may suppose that $h$ is negative everywhere.

We have

$$
\left\{\begin{array}{cc}
-\Delta\left(u^{2}-h\right)=2 \lambda u^{2} e^{u^{2}} & \text { in } \Omega, \\
u^{2}-h=0 & \text { on } \partial \Omega .
\end{array} .\right.
$$

We divide up the right-hand side as

$$
2 \lambda u^{2} e^{u^{2}}=f_{1}+f_{2},
$$

where $f_{1} \in L^{1}$ with $\left\|f_{1}\right\|_{1}<\varepsilon$ and $f_{2} \in L^{\infty}$. Then the solution $w$ for

$$
\left\{\begin{array}{cc}
-\Delta w=f_{2} & \text { in } \Omega \\
w=0 & \text { on } \partial \Omega
\end{array}\right.
$$

belongs to $L^{\infty}$. 
We have

$$
\left\{\begin{array}{cc}
-\Delta\left(u^{2}-h-w\right)=f_{1} & \text { in } \Omega, \\
u^{2}-h-w=0 & \text { on } \partial \Omega
\end{array}\right.
$$

with $\left\|f_{1}\right\|_{1}<\varepsilon$. By virtue of Lemma 4.2 , this implies

$$
e^{u^{2}-h-w} \in L^{4 \pi / \varepsilon} .
$$

Because $h \leq 0$ and $w \in L^{\infty}$,

$$
e^{u^{2}} \in L^{1+\delta}
$$

with some $\delta>0$ if $\varepsilon>0$ is taken to be sufficiently small. Therefore, for $\delta^{\prime} \in(0, \delta)$ we have

$$
\lambda u e^{u^{2}} \in L^{1+\delta^{\prime}}
$$

and a bootstrap argument tells us that $u \in L^{\infty}$.

4.2. Relations between the variational solutions. Adimurthi [1] constructed a variational solution via the Nehari critical point theory. That is, finding a minimizer of

under the constraint

$$
J_{\lambda}(v)=\frac{1}{2}\|\nabla v\|_{2}^{2}-\frac{\lambda}{2} \int_{\Omega}\left(e^{v^{2}}-1\right) d x
$$

$$
\|\nabla u\|_{2}^{2}=\lambda \int_{\Omega} u^{2} e^{u^{2}} d x>0
$$

he invoked P.L. Lions' semi-compactness argument ([11]).

Proposition $4.3([1])$. For all $0<\lambda<\lambda_{1}$, there is a minimizer of $J_{\lambda}$ on $H_{0}^{1}(\Omega)$ with (4.1), denoted by $u$, where $\lambda_{1}$ is the first eigenvalue of $-\Delta$ on $\Omega$ with 0 -Dirichlet boundary condition. It solves (1.1) weakly, and also

$$
0<J_{\lambda}(u)<2 \pi \text {. }
$$

M.-C. Shaw [21] considered a different kind of variational problem. Let

$$
I(v)=\int_{\Omega}\left(e^{v^{2}}-1\right) d x
$$

for $v \in H_{0}^{1}(\Omega)$, and set

$$
\mathcal{S}_{\mu}=\left\{v \in H_{0}^{1}(\Omega) \mid I(v)=\mu\right\}
$$

where $\mu>0$.

Proposition $4.4([21])$. There exists a minimizer $u$ of $E(v)=\|\nabla v\|_{2}^{2}$ on $\mathcal{S}_{\mu}$, provided that

$$
\mathcal{S}_{\mu} \cap\left\{\|\nabla v\|_{2}^{2}<4 \pi\right\} \neq \emptyset
$$

Because $\|\nabla u\|_{2}^{2}<4 \pi$, it solves (1.1) classically with Lagrange multiplier $\lambda$.

Definition. We say that $u$ is the Shaw solution if and only if it minimizes $E(v)$ on $\mathcal{S}_{\mu}$ for some $\mu>0$.

We also formulate the "dual" form of the above.

Definition. We say that $u$ is the dual Shaw solution whenever it maximizes $I(v)$ on $H_{0}^{1}(\Omega)$ under the constraint $\|\nabla v\|_{2}^{2}=E$.

We can prove the following easily. 
Proposition 4.5. For any given $E$ with $0<E<4 \pi$, there exists a maximizer of $I(v)$ on $H_{0}^{1}(\Omega)$ under the constraint $\|\nabla v\|_{2}^{2}=E$.

The results by L. Carleson and S.-Y.A. Chang [4] and by M. Flucher [7] ensure that the dual Shaw solution exists even for $E=4 \pi$. Furthermore, sharpness of (1.2) indicates its non-existence for $E>4 \pi$.

Now we show that those two formulations are equivalent.

Proposition 4.6. Any Shaw solution u maximizes $I(v)$ on $H_{0}^{1}(\Omega)$ under the constraint $\|\nabla v\|_{2}^{2}=\|\nabla u\|_{2}^{2}$. Conversely, any dual Shaw solution $u^{*}$ minimizes $E(v)$ on $H_{0}^{1}(\Omega)$ under the constraint $I(v)=I\left(u^{*}\right)$.

Proof. Let $u$ be the Shaw solution. Then $v \in H_{0}^{1}(\Omega)$ with $I(v)=I(u)$ satisfies $\|\nabla v\|_{2}^{2} \geq\|\nabla u\|_{2}^{2}$. Multiplying by positive constants, we see that the same holds for any $v \in H_{0}^{1}(\Omega)$ with $I(v) \geq I(u)$. Therefore, $v \in H_{0}^{1}(\Omega)$ with $\|\nabla v\|_{2}^{2}<\|\nabla u\|_{2}^{2}$ must satisfy $I(v)<I(u)$. Again by multiplication, $\|\nabla v\|_{2}^{2}=\|\nabla u\|_{2}^{2}$ for $v \in H_{0}^{1}(\Omega)$ implies $I(v) \leq I(u)$.

The latter part follows similarly.

The above proposition indicates that the Shaw solution $u$ satisfies $\|\nabla u\|_{2}^{2} \leq 4 \pi$. We proceed to the relation between those solutions and Adimurthi's. In fact, any Shaw or dual Shaw solution $u$ solves (1.1) classically with some multiplier $\lambda>0$, provided $\|\nabla u\|_{2}^{2}<4 \pi$.

Proposition 4.7. Let $u$ be the solution to (1.1) in the sense of either the Shaw or the dual Shaw formulation. Then it attains the minimum of $J_{\lambda}(v)$ on $H_{0}^{1}(\Omega)$ under the constraint (4.1), i.e., it coincides with the solution of Proposition 4.3.

Proof. We prove the Shaw case. The dual Shaw case follows from the previous proposition.

Let $u$ be the Shaw solution with multiplier $\lambda>0$. Any $v \in H_{0}^{1}(\Omega)$ admits a constant $t>0$ such that $w=t^{-1} v$ solves

$$
\int_{\Omega}\left(e^{w^{2}}-1\right) d x=\int_{\Omega}\left(e^{u^{2}}-1\right) d x .
$$

This implies $\|\nabla u\|_{2}^{2} \leq\|\nabla w\|_{2}^{2}$, and hence

$$
\begin{aligned}
J_{\lambda}(w) & =\frac{1}{2}\|\nabla w\|_{2}^{2}-\frac{\lambda}{2} \int_{\Omega}\left(e^{w^{2}}-1\right) d x \\
& \geq \frac{1}{2}\|\nabla u\|_{2}^{2}-\frac{\lambda}{2} \int_{\Omega}\left(e^{u^{2}}-1\right) d x \\
& =J_{\lambda}(u) .
\end{aligned}
$$

On the other hand, whenever $v$ solves (1.3) we have

$$
J_{\lambda}(v) \geq J_{\lambda}\left(t^{-1} v\right)
$$

for $t>0$. Therefore, $u$ minimize $J_{\lambda}$ on $H_{0}^{1}(\Omega)$ under the constraint (1.3).

Although Carleson and Chang [4] proved that

$$
T_{0}=\sup \left\{\int_{D}\left(e^{v^{2}}-1\right) d x \mid v \in H_{0}^{1}(D),\|\nabla v\|_{2}^{2} \leq 4 \pi\right\}
$$


is attained, McLeod and Peletier [12] gave an alternative proof by the method of ordinary differential equations. Their proof proceeds as follows. First, any $\beta \in(0,1)$ admits a maximizer $v_{\beta}(x)>0$ for

$$
T_{\beta}=\sup \left\{\int_{D}\left(e^{\beta v^{2}}-1\right) d x \mid v \in H_{0}^{1}(D),\|\nabla v\|_{2}^{2} \leq 4 \pi\right\} .
$$

Then, $u=\sqrt{\beta} v_{\beta}$ solves (1.1) with some $\lambda>0$. If $T_{0}$ is not attained, such a family $\{(u, \lambda)\}$ satisfies

$$
\lambda \searrow 0,\|u\|_{\infty} \rightarrow+\infty,\|\nabla u\|_{2}^{2} \nearrow 4 \pi
$$

Then the ODE analysis described above gives us

$$
I=\int_{D}\left(e^{u^{2}}-1\right) d x \rightarrow \pi e .
$$

This implies $T_{0} \leq \pi e$, but $T_{0}>\pi e$ is assured by a function found in [4].

The above relation (Proposition 4.7) between variational solutions is valid for general nonlinearity admitting the Nehari formulation. However, the converse does not necessarily hold. For example, we have the following for the radially symmetric case.

Proposition 4.8. In the case of $\Omega=D$, any family of Shaw or dual Shaw solutions cannot approach $\lambda=0$.

Proof. First, suppose the existence of a family of dual Shaw solutions $\{(u, \lambda)\}$ with $\lambda \searrow 0$. From the definition, $u$ satisfies $\|\nabla u\|_{2}^{2} \leq 4 \pi$; and $\|\nabla u\|_{2}^{2} \rightarrow 4 \pi$ follows as $\lambda \searrow 0$ from (1.7) in Proposition 1.1. Therefore, if the assertion is not true, (4.3) holds and hence (4.4) also.

However, $u$ solves the dual Shaw problem, so that

$$
\int_{D}\left(e^{v^{2}}-1\right) d x \leq \int_{D}\left(e^{u^{2}}-1\right) d x
$$

for any $v \in H_{0}^{1}(D)$ with $\|\nabla v\|_{2}^{2} \leq\|\nabla u\|_{2}^{2}$. This implies that

$$
\begin{aligned}
T_{0} & =\sup \left\{\int_{D}\left(e^{v^{2}}-1\right) d x \mid\|\nabla v\|_{2}^{2} \leq 4 \pi\right\} \\
& \leq \varliminf_{\lambda \rightarrow 0} \int_{D}\left(e^{u^{2}}-1\right) d x=\pi e .
\end{aligned}
$$

This is a contradiction, as indicated.

Next, suppose that a family of Shaw solutions $\{(u, \lambda)\}$ exists until $\lambda \searrow 0$. Then, Theorem 1.2 implies $\|\nabla u\|_{2}^{2} \rightarrow 4 \pi$. As described after Proposition 4.6, this means that $\|\nabla u\|_{2}^{2} \nearrow 4 \pi$ and $\{(u, \lambda)\}$ is a family of dual Shaw solutions. Therefore, $\lambda \searrow 0$ is impossible from the previous argument.

In connection with this, the following fact should be noted. For the supercritical problem

$$
\left\{\begin{array}{cc}
-\Delta u=\lambda f(u) e^{u^{2}}, u>0 & \text { in } D \\
u=0 & \text { on } \partial D
\end{array}\right.
$$

with $\lim _{u \rightarrow+\infty} f(u) / u=+\infty$, it is possible to construct a family of solutions $\{(u, \lambda)\}$ to $(4.5)$ satisfying (4.3) under the Shaw formulation ([18]). 
In spite of Proposition 4.8, the asymptotics (4.4) holds as $\lambda \searrow 0$. This follows on inspecting McLeod and Peletier [12] carefully to see that (4.4) is a consequence of only

$$
\|u\|_{\infty} \rightarrow+\infty, \quad\|\nabla u\|_{2}^{2} \rightarrow 4 \pi, \quad \lambda \searrow 0 .
$$

\section{Simply Connected Domains}

5.1. Global behavior. We return to the problem (1.1) but on the bounded simply connected domain $\Omega$. Namely, the present section is devoted to the proof of Theorem 1.5 and Proposition 1.6. Those results can be extended to other nonlinearities by slight modifications.

Lemma 5.1. Let $\Omega \subset \mathbb{R}^{2}$ be a simply connected domain with a smooth boundary and $u$ a classical solution of

$$
\left\{\begin{array}{cc}
-\Delta u=f(u), u>0 & \text { in } \Omega, \\
u=0 & \text { on } \partial \Omega,
\end{array}\right.
$$

where $f \in C^{1}(\mathbb{R}, \mathbb{R})$ with $f(u) \geq 0(u \geq 0)$. Then

$$
2 \int_{\Omega} F(u) d x \leq \frac{1}{4 \pi}\left(\int_{\Omega} f(u) d x\right)^{2} \leq C_{\Omega} \int_{\Omega} F(u) d x
$$

with a constant $C_{\Omega}>0$ determined only by $\Omega$, where

$$
F(u)=\int_{0}^{u} f(u) d u .
$$

Proof. The first inequality of (5.2) is due to L.Payne, R. Sperb and I. Stakgold [19] (cf. Sperb [22]). To prove the second inequality, we prepare a conformal mapping $H: D \rightarrow \Omega$. Note that $H^{\prime}$ and $H^{\prime \prime}$ are bounded. Setting $w=H^{-1} u$ and $h(x)=\left|H^{\prime}(z)\right|^{2}$, we transform (5.1) into

$$
\left\{\begin{array}{cc}
-\Delta w=h(x) f(w) & \text { in } D, \\
w=0 & \text { on } \partial D .
\end{array}\right.
$$

Multiply (5.3) by $x \cdot \nabla w$ and integrate by parts to obtain

$$
\begin{gathered}
\int_{D}(2 h(x) F(w)+(x \cdot \nabla h) F(w)) d x \\
=\frac{1}{2} \int_{\partial D}(x \cdot n)|\nabla w|^{2} d s
\end{gathered}
$$

where $n$ denotes the unit outer normal vector.

Here, noting that $\int_{\partial D} \frac{d s}{x \cdot n}=2 \pi$, we utilize Schwarz' inequality to deduce that

$$
\begin{aligned}
\int_{\partial D}(x \cdot n)|\nabla w|^{2} d x & =\int_{\partial D}\left(\frac{\partial w}{\partial n}\right)^{2} x \cdot n d s \\
& \geq\left(\int_{\partial D}-\frac{\partial w}{\partial n} d s\right)^{2} / \int_{\partial D} \frac{d s}{x \cdot n} \\
& =\frac{1}{2 \pi}\left(\int_{D} h(x) f(w) d x\right)^{2} \\
& =\frac{1}{2 \pi}\left(\int_{\Omega} f(u) d x\right)^{2}
\end{aligned}
$$


On the other hand, for $\kappa(x)=x \cdot \nabla \log h(x)$ we have

$$
\begin{aligned}
\int_{D}(x \cdot \nabla h) F(w) d x & \leq \max _{x \in \bar{D}} \kappa(x) \int_{D} F(w) h(x) d x \\
& =\max _{x \in \bar{D}} \kappa(x) \int_{\Omega} F(u) d x .
\end{aligned}
$$

Therefore,

$$
\frac{1}{4 \pi}\left(\int_{\Omega} f(u) d x\right)^{2} \leq\left(2+\max _{x \in \bar{D}} \kappa(x)\right) \int_{\Omega} F(u) d x .
$$

Applying the above lemma to (1.1), we have

$$
4 \pi \lambda \int_{\Omega}\left(e^{u^{2}}-1\right) \leq\left(\lambda \int_{\Omega} u e^{u^{2}} d x\right)^{2} \leq \sigma_{\Omega} \lambda \int_{\Omega}\left(e^{u^{2}}-1\right) d x
$$

with $\sigma_{\Omega}=2 \pi C_{\Omega}$.

Now we can prove (1.10)-(1.12). In fact, the first relation (1.10) was proved in section 1. For the second inequality, we make use of (5.5); thus, for any $t>0$,

$$
\begin{aligned}
\left(\lambda \int_{\Omega} u e^{u^{2}} d x\right)^{2} & \leq \sigma_{\Omega} \lambda\left\{\int_{\{u>t\}}\left(e^{u^{2}}-1\right) d x+\int_{\{u \leq t\}}\left(e^{u^{2}}-1\right) d x\right\} \\
& \leq \frac{\sigma_{\Omega}}{t} \lambda \int_{\{u>t\}} u e^{u^{2}} d x+\lambda \sigma_{\Omega}|\Omega|\left(e^{t^{2}}-1\right) \\
& \leq \frac{\sigma_{\Omega}}{t} \lambda \int_{\Omega} u e^{u^{2}} d x+\lambda \sigma_{\Omega}|\Omega|\left(e^{t^{2}}-1\right) .
\end{aligned}
$$

Therefore,

$$
\varlimsup_{\lambda \rightarrow 0} \lambda \int_{\Omega} u e^{u^{2}} d x \leq \frac{\sigma_{\Omega}}{t},
$$

which implies the desired inequality. The third relation is a consequence of the second one and (5.5).

5.2. Single point blow-up. The proof of (1.14) and (1.15) requires the following.

Lemma 5.2. Under the assumption (1.13), we have

$$
\lim _{\lambda \rightarrow 0} \int_{\bar{\omega}} u^{p} d x=0
$$

for any $\bar{\omega} \subset \subset \Omega$ and $1 \leq p<\infty$.

Proof. In fact, taking the first eigenfunction $\phi_{1}>0$ of $-\Delta$ with the homogeneous boundary condition, we have

$$
\lambda_{1} \int_{\Omega} \phi_{1} u d x=\lambda \int_{\Omega} \phi_{1} u e^{u^{2}} d x \leq C \lambda \int_{\Omega} u e^{u^{2}} d x \rightarrow 0
$$

as $\lambda \searrow 0$. This shows that $\int_{\bar{\omega}} u d x \rightarrow 0$. Then, the Gagliardo-Nierenberg inequality (cf. Brezis [2]) implies

$$
\left(\int_{\bar{\omega}} u^{p} d x\right)^{1 / p} \leq C\|u\|_{L^{1}(\bar{\omega})}^{1-a}\|\nabla u\|_{2}^{a} \rightarrow 0
$$

for $1<p<\infty$ and $a=1-\frac{1}{p}>0$. 
In particular the above lemma implies $\|u\|_{L^{1}(\bar{\omega})}=O(1)$ for any $\bar{\omega} \subset \subset \Omega$. Then the argument of P.G. de Figueiredo, P.L. Lions and R. Nussbaum [6] based on an assertion in B. Gidas, Ni W.-M. and L. Nirenberg ([8], p.223) assures that $\mathcal{S} \subset \subset \Omega$.

Let $x \in \mathcal{S}$ and $\delta>0$ be so that $2 \delta<d(x, \partial \Omega)$. We suppose, contrary to (1.14), that

$$
\varlimsup_{\lambda \rightarrow 0} \int_{D_{2 \delta}\left(x_{0}\right)}|\nabla u|^{2} d x<4 \pi .
$$

We introduce a cut-off function $\phi_{\delta}(\cdot)=\phi\left(\frac{\cdot-x}{\delta}\right)$, where $\phi \in C_{0}^{\infty}\left(\mathbb{R}^{2}\right)$ with $\phi(x)=0$ for $|x| \geq 2$ and $\phi(x)=1$ for $|x| \leq 1$. Then, for small $\varepsilon>0$,

$$
\begin{aligned}
\left\|\nabla\left(\phi_{\delta} u\right)\right\|_{2}^{2} & \leq(1+\varepsilon) \int_{\Omega} \phi_{\delta}^{2}|\nabla u|^{2} d x+\left(1+\frac{1}{\varepsilon}\right) \int_{\Omega} u^{2}\left|\nabla \phi_{\delta}\right|^{2} d x \\
& \leq(1+\varepsilon) \int_{D_{2 \delta}\left(x_{0}\right)}|\nabla u|^{2}+C\left(1+\frac{1}{\varepsilon}\right) \delta^{-2} \int_{D_{2 \delta}\left(x_{0}\right) \backslash D_{\delta}\left(x_{0}\right)} u^{2} d x,
\end{aligned}
$$

so that

$$
\varlimsup_{\lambda \rightarrow 0}\left\|\nabla\left(\phi_{\delta} u\right)\right\|_{2}^{2}<4 \pi .
$$

The localized equation is given as

$$
\left\{\begin{array}{cc}
-\Delta\left(\phi_{\delta / 2} u\right)=\lambda \phi_{\delta / 2} u e^{u^{2}}-F & \text { in } D_{\delta}\left(x_{0}\right), \\
\phi_{\delta / 2} u=0 & \text { on } \partial D_{\delta}\left(x_{0}\right),
\end{array}\right.
$$

where $F=2 \nabla u \cdot \nabla \phi_{\delta / 2}+u \Delta \phi_{\delta / 2} \in L^{2}\left(D_{\delta}\left(x_{0}\right)\right)$. Here, from (5.7) and the TrudingerMoser inequality it follows that

$$
\lambda \phi_{\delta / 2} u e^{u^{2}} \in L^{1+\eta}\left(D_{\delta}\left(x_{0}\right)\right)
$$

with some $\eta>0$. Then, an elliptic regularity estimate implies

$$
\|u\|_{L^{\infty}\left(D_{\delta / 2}\left(x_{0}\right)\right)}=O(1)
$$

(see Gilbarg-Trudinger [9]), contradicting $x_{0} \in \mathcal{S}$. This shows (1.14).

Finally, any compact set $\bar{\omega} \subset \subset \Omega \backslash \mathcal{S}$ admits a constant $C>0$ so that $\|u\|_{L^{\infty}(\bar{\omega})} \leq$ $C$. Hence by (1.1),

$$
\|\Delta u\|_{L^{\infty}(\bar{\omega})} \leq C^{\prime}
$$

with another constant $C^{\prime}>0$. Combined with Lemma 5.2, this implies $\|u\|_{L^{\infty}(\bar{\omega})} \rightarrow$ 0 as $\lambda \searrow 0$, or (1.15) by the Poincaré-Wirtinger inequality (see, e.g., Brezis [2]).

We finally give the proof of Proposition 1.6.

Proof of Proposition 1.6. As we have shown above, the blow-up points are necessarily in the interior. The relation (1.16) is a direct consequence of (4.2) and the third relation of (1.7). In fact, we have

$$
4 \pi \leq \varliminf_{\lambda \rightarrow 0}\|\nabla u\|_{2}^{2} \leq \varlimsup_{\lambda \rightarrow 0}\|\nabla u\|_{2}^{2}=\varlimsup_{\lambda \rightarrow 0} 2 J_{\lambda}(u) \leq 4 \pi .
$$




\section{ACKNOWLEDGEMENT}

Most of this work was done while the authors were visiting Johns Hopkins University in March 1994. They thank Professors Hiroshi Matano and Joel Spruck for invitation to the JAMI conference, and the Department of Mathematics for hospitality. The first author is grateful to Professors Ni Wei-Ming and Michael Struwe for stimulating discussions.

\section{REFERENCES}

[1] Adimurthi, Existence of positive solutions of the semi-linear Dirichlet problem with critical growth for the n-Laplacian, Ann. Scuola Norm. Sup. Pisa IV 17 (1990), 393-413. MR 91j:35016

[2] H. Brézis, Analyse Fonctionnelle, Théorie et Applications, Masson, Paris, 1983. MR 85a: 46001

[3] H. Brézis and F. Merle, Uniform estimate and blow-up behavior for solutions of $-\Delta u=$ $V(x) e^{u}$ in two dimensions, Comm. Partial Differential Equations 16 (1991), 1223-1253. MR 92m:35084

[4] L. Carleson and S-Y. A. Chang, On the existence of an extremal function for an inequality of J.Moser, Bull. Sci. Math. 110 (1986), 113-127. MR 88f:46070

[5] W.-X. Chen and C.-M. Li, Classification of solutions of some nonlinear elliptic equations, Duke Math. J. 63 (1991), 615-622. MR 93e:35009

[6] P.G. De Figueiredo, P.L. Lions and R.D. Nussbaum, A priori estimates and existence of positive solutions of semi-linear elliptic equations, J. Math. Pures Appl. 61 (1982), 41-63. MR 83h:35039

[7] M. Flucher, Extremal functions for the Trudinger-Moser inequality in 2 dimensions, Comment. Math. Helv. 67 (1992), 471-497. MR 93k:58073

[8] B. Gidas, W.-M. Ni and L. Nirenberg, Symmetry and related properties via the maximum principle, Comm. Math. Phys. 68 (1979), 209-243. MR 80h:35043

[9] D. Gilbarg and N. Trudinger, Elliptic Partial Differential Equations of Second Order, 2nd ed., Springer, Berlin, 1983. MR 86c:35035

[10] T. Itoh, Blowing up behavior of solutions of nonlinear elliptic equations, Spectral and Scattering Theory and Applications (K. Yajima ed.), Adv. Stud. Pure Math. 23, Birkhäuser 1994, pp. 177-186. MR 95e:35074

[11] P.L. Lions, The concentration compactness principle in the calculus of variations, the limit case, Riv. Mat. Iberoamericana, 1 (1985), 145-201. MR 87c:49007

[12] J.B. McLeod and L.A. Peletier, Observations on Moser's inequality, Arch. Rat. Mech. Anal. 106 (1988), 261-285. MR 90d:26029

[13] J. Moser, A sharp form of an inequality by N.Trudinger, Indiana Univ. Math. J. 11 (1971), 1077-1092. MR 46:662

[14] Z. Nehari, On a class of nonlinear second-order differential equations, Trans. Amer. Math. Soc. 95 (1960), 101-123. MR 22:2756

[15] T. Ogawa, A proof of Trudinger's inequality and its application to nonlinear Schrödinger equations, Nonlinear Anal. 14 (1990), 765-769. MR 91d:35203

[16] T. Ogawa and T. Suzuki, Trudinger's inequality and related nonlinear elliptic equations in two dimensions, Spectral and Scattering Theory and Applications (K. Yajima ed.), Adv. Stud. Pure Math. 23, Birkhäuser 1994, pp.283-294. MR 95e:35077

[17] T. Ogawa and T. Suzuki, Nonlinear elliptic equations with critical growth related to the Trudinger inequality, Asymptotic Analysis, 12 (1996), 25-40. CMP 96:08

[18] T. Ogawa and T. Suzuki, Microscopic asymptotics for solutions of some elliptic equations, Nagoya Math. J. 138 (1995), 33-50. MR 96e:35040

[19] L.E. Payne, R.P. Sperb, and I. Stakgold, On Hopf type maximum principles for convex domains, Nonlinear Anal. 1 (1977), 547-559. MR 80m:35026

[20] S.I. Pohozaev, Eigenfunctions of the equation $\Delta u+\lambda f(u)=0$, Dokl. Akad. Nauk SSSR 165 (1965), 36-39; English transl., Soviet Math. Dokl. 6 (1965), 1408-1411. MR 33:411

[21] M.-C. Shaw, Eigenfunctions of the nonlinear equation $\Delta u+\nu f(x, u)=0$ in $R^{2}$, Pacific J. Math. 129 (1987), 349-356. MR 88k:35158 
[22] R. Sperb, Maximum Principles and Their Applications, Academic Press, New York, 1981. MR 84a:35033

[23] M. Struwe, Critical points of embeddings of $H_{0}^{1, n}$ into Orlicz spaces, Ann. Inst. Henri Poincáre, Analyse Non Linéaire 5 (1988), 425-464. MR 90c:35084

[24] N.S. Trudinger, On imbedding into Orlicz space and some applications, J. Math. Mech. 17 (1967), 473-484. MR 35:7121

Department of Mathematics, University of California at Santa Barbara, Santa BarBara, California 93106

Current address: Graduate School of Mathematics, Kyushu University 36, Fukuoka, 812-8581, Japan

E-mail address: ogawa@math.kyushu-u.ac.jp

Department of Mathematics, Osaka University, Toyonaka, Osaka 560, Japan

E-mail address: takashi@math.sci.osaka-u.ac.jp 